IJMMS 29:8 (2002) 481-494

PII. S0161171202012206

http://ijmms.hindawi.com

(c) Hindawi Publishing Corp.

\title{
GENERALIZED SOLUTIONS DESCRIBING SINGULARITY INTERACTION
}

\author{
V. G. DANILOV
}

Received 28 February 2001

We present a new method for constructing solutions to nonlinear evolutionary equations describing the propagation and interaction of nonlinear waves.

2000 Mathematics Subject Classification: 35L60, 58J47.

1. Introduction. The goal of the present paper is to demonstrate a new approach to the construction of asymptotic solutions to nonlinear evolutionary equations, which we call the weak asymptotics method.

Usually, by saying that a function is an asymptotic (approximate) solution of a differential equation, we mean that this function satisfies the equation with a small discrepancy. The smallness of the discrepancy is understood as the smallness in some uniform metric under the assumption that a small parameter tends to zero.

A function is called a weak asymptotic solution if, after the substitution of this function into the equation, there is a discrepancy that is small in the weak sense as a small parameter tends to zero. In this case the functionals are assumed to depend on time as on a parameter.

For example, under this approach, the $C^{\infty}$-approximation of a generalized function turns out to be its weak asymptotics and we can choose generalized functions to be the initial conditions and use their approximations for constructing the solutions. In this case, we obtain a small parameter, which is either the parameter of approximation or the small parameter in the original equation. In the latter case, this original small parameter is taken to be the parameter of approximation.

In fact, this approach is close to the ideas proposed by Colombeau and other authors who constructed different algebras of generalized functions. The difference is that in our approach the mollifier is chosen not from the consideration of the algebraic construction but from the consideration of the original differential equation.

In some cases (shock waves), the solution is independent of the choice of the mollifier, while in other cases (solitons, kinks) the solution depends on this choice.

If the original equation contains a small parameter, then we, in fact, deal with regularizations by small viscosity or small dispersion. In this case, to calculate a weak asymptotics, we need to calculate the zero viscosity and zero dispersion limits. Hence we arrive at the problem of constructing a definition of a weak solution which admits this passage to the limit. 
It turned out that the approach developed here can be used for describing both the propagation of nonlinear waves and, which is the most important, their interaction. In what follows, we consider the main technical tools and some examples which allow us to demonstrate the abilities of our approach.

\section{Some weak asymptotic formulas}

2.1. Let $\omega(z) \in \mathbb{S}\left(\mathbb{R}^{1}\right)$, where $\mathbb{S}$ is the Schwartz space. We consider the function $(1 / \varepsilon) \omega((x-a) / \varepsilon)$ and calculate its weak asymptotics. Treating $(1 / \varepsilon) \omega((x-a) / \varepsilon)$ as a generalized function, for any function $\eta(x) \in C_{0}^{\infty}$ we have

$$
\begin{aligned}
\left\langle\frac{1}{\varepsilon} \omega\left(\frac{x-a}{\varepsilon}\right), \eta(x)\right\rangle & =\frac{1}{\varepsilon} \int \omega\left(\frac{x-a}{\varepsilon}\right) \eta(x) d x=\int \omega(z) \eta(a+\varepsilon z) d z \\
& =\sum_{k \geq 0} \Omega_{k} \frac{\varepsilon^{k}}{k !}(-1)^{k}\left\langle\delta^{(k)}(x-a), \eta\right\rangle, \quad \varepsilon>0,
\end{aligned}
$$

where the last relation is formal and means that the left-hand side can be represented as the asymptotic series given on the right-hand side,

$$
\Omega_{k}=\int \omega(z) z^{k} d z \text {. }
$$

We define by $O_{\mathscr{D}^{\prime}}\left(\varepsilon^{\alpha}\right)$ an element of $\mathscr{D}^{\prime}$ such that

$$
f(x, \varepsilon)=O_{\mathscr{D}^{\prime}}\left(\varepsilon^{\alpha}\right) \Longleftrightarrow\langle f(x, \varepsilon), \eta(x)\rangle=O\left(\varepsilon^{\alpha}\right),
$$

where the last $O$-estimate (which must hold for any function $\eta(x) \in C_{0}^{\infty}$ ) is understood in the usual sense. Then for any $N$ we can write

$$
\frac{1}{\varepsilon} \omega\left(\frac{x-a}{\varepsilon}\right)=\sum_{k=0}^{N} \Omega_{k} \frac{\varepsilon^{k}}{k !}(-1)^{k} \delta^{(k)}(x-a)+O_{\Phi^{\prime}}\left(\varepsilon^{N+1}\right) .
$$

2.2. Let $\omega_{1}(z), \omega_{2}(z) \in \mathbb{S}\left(\mathbb{R}^{1}\right)$. Consider the weak asymptotics of the product $\omega_{1}\left(\left(x-a_{1}\right) / \varepsilon\right) \omega_{2}\left(\left(x-a_{2}\right) / \varepsilon\right)$. We have

$$
\begin{aligned}
\left\langle\omega_{1}\left(\frac{x-a_{1}}{\varepsilon}\right) \omega_{2}\left(\frac{x-a_{2}}{\varepsilon}\right), \eta(x)\right\rangle & =\int \omega_{1}\left(\frac{x-a_{1}}{\varepsilon}\right) \omega_{2}\left(\frac{x-a_{2}}{\varepsilon}\right) \eta(x) d x \\
& =\varepsilon \eta\left(a_{1}\right) \int \omega_{1}(z) \omega_{2}\left(z-\frac{\Delta a}{\varepsilon}\right) d z+O\left(\varepsilon^{2}\right) \\
& =\varepsilon \eta\left(a_{2}\right) \int \omega_{1}\left(z+\frac{\Delta a}{\varepsilon}\right) \omega_{2}(z) d z+O\left(\varepsilon^{2}\right), \\
\Delta a & =a_{2}-a_{1} .
\end{aligned}
$$

Finally, we obtain the following formula that is uniform and symmetric in $a_{1}, a_{2}$ :

$$
\omega_{1}\left(\frac{x-a_{1}}{\varepsilon}\right) \omega_{2}\left(\frac{x-a_{2}}{\varepsilon}\right)=\frac{1}{2}\left[\varepsilon \delta\left(x-a_{1}\right)+\varepsilon \delta\left(x-a_{2}\right)\right] B\left(\frac{\Delta a}{\varepsilon}\right)+O_{\mathscr{D}^{\prime}}\left(\varepsilon^{2}\right),
$$

where

$$
B\left(\frac{\Delta a}{\varepsilon}\right)=\int \omega_{1}(z) \omega_{2}\left(z-\frac{\Delta a}{\varepsilon}\right) d z=\int \omega_{1}\left(z+\frac{\Delta a}{\varepsilon}\right) \omega_{2}(z) d z
$$


2.3. Now let $\omega_{1}(z), \omega_{2}(z) \in C^{\infty}, d \omega_{i} / d z \in \mathbb{S}\left(\mathbb{R}^{1}\right), \lim _{z \rightarrow-\infty} \omega_{i}=0, \lim _{z \rightarrow \infty} \omega_{i}=1$, $i=1,2$.

Calculate the weak asymptotics of the derivative

$$
\begin{aligned}
\frac{d}{d x} \omega_{1}\left(\frac{x-a_{1}}{\varepsilon}\right) \omega_{2}\left(\frac{x-a_{2}}{\varepsilon}\right)= & \frac{1}{\varepsilon} \dot{\omega}_{1}\left(\frac{x-a_{1}}{\varepsilon}\right) \omega_{2}\left(\frac{x-a_{2}}{\varepsilon}\right) \\
& +\frac{1}{\varepsilon} \omega_{1}\left(\frac{x-a_{1}}{\varepsilon}\right) \dot{\omega}_{2}\left(\frac{x-a_{2}}{\varepsilon}\right) .
\end{aligned}
$$

Just as previously, we have

$$
\begin{aligned}
\frac{1}{\varepsilon} \dot{\omega}_{1}\left(\frac{x-a_{1}}{\varepsilon}\right) & \omega_{2}\left(\frac{x-a_{2}}{\varepsilon}\right)+\frac{1}{\varepsilon} \omega_{1}\left(\frac{x-a_{1}}{\varepsilon}\right) \dot{\omega}_{2}\left(\frac{x-a_{2}}{\varepsilon}\right) \\
= & \delta\left(x-a_{1}\right) B_{1}\left(\frac{\Delta a}{\varepsilon}\right)+\delta\left(x-a_{2}\right) B_{2}\left(\frac{\Delta a}{\varepsilon}\right)+O_{\mathscr{D}^{\prime}}(\varepsilon),
\end{aligned}
$$

where

$$
B_{1}\left(\frac{\Delta a}{\varepsilon}\right)=\int \dot{\omega}_{1}(z) \omega_{2}\left(z-\frac{\Delta a}{\varepsilon}\right) d z, \quad B_{2}\left(\frac{\Delta a}{\varepsilon}\right)=\int \omega_{1}\left(z+\frac{\Delta a}{\varepsilon}\right) \dot{\omega}_{2}(z) d z .
$$

We have

$$
B_{1}(\infty)=0, \quad B_{1}(-\infty)=1, \quad B_{1}(z)+B_{2}(z) \equiv 1 .
$$

Calculating the primitive, we obtain

$$
\omega_{1}\left(\frac{x-a_{1}}{\varepsilon}\right) \omega_{2}\left(\frac{x-a_{2}}{\varepsilon}\right)=\theta\left(x-a_{1}\right) B_{1}\left(\frac{\Delta a}{\varepsilon}\right)+\theta\left(x-a_{2}\right) B_{2}\left(\frac{\Delta a}{\varepsilon}\right)+O_{\mathscr{D}^{\prime}}(\varepsilon) .
$$

2.4. Under the assumptions of item (b) and the condition that $\int \omega_{i}(z) d z=1$, the functions $\omega_{i}\left(\left(x-a_{i}\right) / \varepsilon\right.$ ) are approximations (weak asymptotics) of the functions $\varepsilon \delta\left(x-a_{i}\right)$,

$$
\omega_{i}\left(\frac{x-a_{i}}{\varepsilon}\right)=\varepsilon \delta_{\varepsilon, i}\left(x-a_{i}\right)
$$

Hence we can rewrite (2.6) as

$$
\varepsilon \delta_{\varepsilon, 1}\left(x-a_{1}\right) \varepsilon \delta_{\varepsilon, 2}\left(x-a_{2}\right)=\frac{1}{2}\left[\varepsilon \delta\left(x-a_{1}\right)+\varepsilon \delta\left(x-a_{2}\right)\right] B\left(\frac{\Delta a}{\varepsilon}\right)+O_{\mathscr{D}^{\prime}}\left(\varepsilon^{2}\right) .
$$

In a similar way, under the assumptions of Section 2.3, $\omega_{i}\left(\left(x-a_{1}\right) / \varepsilon\right)=\theta_{\varepsilon, i}\left(x-a_{i}\right)$ are approximations of the Heaviside $\theta$-function. Hence we can rewrite (2.12) as

$$
\theta_{\varepsilon, 1}\left(x-a_{1}\right) \theta_{\varepsilon, 2}\left(x-a_{2}\right)=\theta\left(x-a_{1}\right) B_{1}\left(\frac{\Delta a}{\varepsilon}\right)+\theta\left(x-a_{2}\right) B_{2}\left(\frac{\Delta a}{\varepsilon}\right)+O_{\mathscr{D}^{\prime}}(\varepsilon) .
$$

3. Nonlinear structures. We show how the above formulas can be used to describe interaction of nonlinear structures. 
3.1. Interaction of shock waves for the Hopf equation. Consider the Cauchy problem

$$
L[u]=u_{t}+\left(u^{2}\right)_{x}=0,\left.\quad u\right|_{t=0}=u_{0}+u_{1} \theta\left(-x+a_{1}\right)+u_{2} \theta\left(-x+a_{2}\right),
$$

where $u_{i}$ are positive constants, $a_{2}<a_{1}$. We approximate the initial condition according to the formulas from Section 2.3 and seek the weak asymptotics of the solution in the form

$$
\begin{gathered}
u_{\varepsilon}(x, t)=u_{0}+u_{1} \theta_{\varepsilon, 1}\left(-x+\varphi_{1}(t, \varepsilon)\right)+u_{2} \theta_{\varepsilon, 2}\left(-x+\varphi_{2}(t, \varepsilon)\right), \\
\varphi_{1}(0)=a_{1}, \quad \varphi_{2}(0)=a_{2} .
\end{gathered}
$$

Calculating the weak asymptotics of the expression $\left(u_{\varepsilon}\right)^{2}$ according to the formulas from Section 2.3, we obtain

$$
\begin{aligned}
\left(u_{\varepsilon}\right)^{2}= & u_{0}^{2}+\left(u_{1}^{2}+2 u_{0} u_{1}\right) \theta\left(-x+\varphi_{1}\right)+u_{0}^{2}+\left(u_{2}^{2}+2 u_{0} u_{2}\right) \theta\left(-x+\varphi_{2}\right) \\
& +2 u_{1} u_{2}\left[\theta\left(-x+\varphi_{1}\right) B_{1}\left(\frac{\Delta \varphi}{\varepsilon}\right)+\theta\left(-x+\varphi_{2}\right) B_{2}\left(\frac{\Delta \varphi}{\varepsilon}\right)\right]+O_{\mathscr{D}^{\prime}}(\varepsilon),
\end{aligned}
$$

where

$$
\begin{aligned}
& B_{1}\left(\frac{\Delta \varphi}{\varepsilon}\right)=\int \dot{\omega}_{1}(z) \omega_{2}\left(z+\frac{\Delta \varphi}{\varepsilon}\right) d z, \\
& B_{2}\left(\frac{\Delta \varphi}{\varepsilon}\right)=\int \omega_{1}\left(z-\frac{\Delta \varphi}{\varepsilon}\right) \dot{\omega}_{2}(z) d z, \quad \Delta \varphi=\varphi_{2}-\varphi_{1},
\end{aligned}
$$

and, in contrast to Section 2.3, we have $B_{1}(-\infty)=0, B_{1}(\infty)=1$, but as before, $B_{1}+$ $B_{2} \equiv 1$.

We substitute the approximation of $u_{\varepsilon}(x, t)$ into the Hopf equation and require that the relation $L\left[u_{\varepsilon}\right]=O_{\mathscr{D}^{\prime}}(\varepsilon)$ must be satisfied (this is the definition of the weak asymptotics solution in this case). Moreover, the function $L\left[u_{\varepsilon}\right]$ must be weakly piecewise continuous with respect to $t$ for each fixed $\varepsilon$. We obtain

$$
L\left[u_{\varepsilon}\right]=\sum_{k=1}^{2}\left[u_{k} \frac{d \varphi_{k}}{d t}-2 u_{0} u_{k}-u_{k}^{2}-2 u_{1} u_{2} B_{k}\left(\frac{\Delta \varphi}{\varepsilon}\right)\right] \delta\left(-x+\varphi_{k}\right)+O_{\mathscr{D}^{\prime}}(\varepsilon) .
$$

Hence, in view of the definition of the weak solution, we have

$$
\frac{d \varphi_{k}}{d t}=2 u_{0}+u_{k}+2 u_{3-k} B_{k}\left(\frac{\Delta \varphi}{\varepsilon}\right), \quad k=1,2 .
$$

For $\Delta \varphi<0$ (before the interaction) we have $B_{1}(\Delta \varphi / \varepsilon)=0$ and $B_{2}(\Delta \varphi / \varepsilon)=1$ up to $O\left(\varepsilon^{N}\right)$ and (3.6) describe the propagation of noninteracting shock waves. We write

$$
\varphi_{10}=\left(2 u_{0}+u_{1}\right) t+a_{1}, \quad \varphi_{20}=\left(2\left(u_{0}+u_{1}\right)+u_{2}\right) t+a_{2},
$$

then $\psi_{0}(t)=\varphi_{20}-\varphi_{10}$ is the distance between the fronts of noninteracting waves. At time $t^{*}, \psi_{0}\left(t^{*}\right)=0$, the fronts merge. To construct a formula that is uniform in $t$ and represents a weak asymptotic solution, we seek the phases $\varphi_{k}(t, \varepsilon)$ of shock waves in the form

$$
\varphi_{k}(t, \varepsilon)=\varphi_{k 0}(t)+\psi_{0} \phi_{k 1}(\tau)
$$


where $\tau=\psi_{0}(t) / \varepsilon$ and it is assumed that

$$
\left.\phi_{k 1}(\tau)\right|_{\tau \rightarrow-\infty}=0,\left.\quad \frac{\phi_{k 1}}{d \tau}\right|_{|\tau| \rightarrow \infty}=o\left(\tau^{-1}\right) .
$$

Calculating the limit values of $\phi_{k 1}(\infty)=\phi_{k 1}^{+}$, we obtain formulas that describe the coordinates of the fronts of shock waves $\varphi_{k}^{+}(t)$ after the interaction. Substituting expressions (3.8) into (3.6), we obtain

$$
\frac{d \varphi_{k 0}}{d t}+\frac{d \psi_{0}}{d t} \frac{d}{d \tau}\left[\tau \varphi_{k 1}(\tau)\right]=2 u_{0}+u_{1}+2 u_{3-k} B_{k}\left(\frac{\Delta \varphi}{\varepsilon}\right), \quad k=1,2 .
$$

We calculate the difference of these equation

$$
\frac{d \rho}{d \tau}=F(\rho), \quad \rho=\frac{\Delta \varphi}{\varepsilon}, F(\rho)=2 B_{2}(\rho)-1 .
$$

The boundary condition for this equation has the form $\rho /\left.\tau\right|_{\tau \rightarrow-\infty} \rightarrow 1$. The equation $F(\rho)=0$ has a single root $\rho_{0}$ and $B_{2}\left(\rho_{0}\right)=B_{1}\left(\rho_{0}\right)=1 / 2$, which implies that after the interaction $\left(\psi_{0}>0, \tau=\psi_{0} / \varepsilon \rightarrow \infty\right)$ the wave fronts move with the same velocity

$$
\frac{d \varphi_{k}^{+}}{d t}=2 u_{0}+u_{1}+u_{2}, \quad k=1,2 .
$$

From (3.6) for the functions $\phi_{k 1}$ we obtain

$$
\phi_{k 1}=(-1)^{k-1} \frac{2 u_{3-k}}{\left(u_{1}+u_{2}\right) \tau} \int_{0}^{\tau}\left[B_{2}(\rho)-1\right] d \tau^{\prime} .
$$

The weak limit $u_{0}(x, t)$ of the weak asymptotic solution $u_{\varepsilon}(x, t)$ satisfies the classical definition of the generalized solution (in the form of integral identity) and the stability condition.

3.2. Interaction of weak discontinuities. Generation and decay of shock waves. We again consider the Hopf equation and pose the following initial condition:

$$
\left.u\right|_{t=0}=u_{0}^{0}+u_{1}^{0}\left(a_{1}-x\right)_{+}-u_{1}^{0}\left(a_{2}-x\right)_{+},
$$

where $a_{1}>a_{2}, z_{+}=z \theta(z), u_{i}^{0}=$ const $>0$ (see Figure 3.1).

We seek the weak asymptotic solution in the form

$$
\begin{aligned}
u_{\varepsilon}(x, t)= & u_{0}+u_{1}\left(\varphi_{1}(t, \varepsilon)-x\right) \theta_{\varepsilon, 1}\left(-x+\varphi_{1}(t, \varepsilon)\right) \\
& -u_{2}\left(\varphi_{2}(t, \varepsilon)-x\right) \theta_{\varepsilon, 2}\left(-x+\varphi_{2}(t, \varepsilon)\right) .
\end{aligned}
$$

In this case, the equations for the functions $u_{i}=u_{i}(t, \varepsilon)$ and $\varphi_{i}=\varphi_{i}(t, \varepsilon)$ are derived by using a somewhat different technique than that used for studying shock waves. Substituting the approximation of $u_{\varepsilon}(x, t)$ into the equation and taking into account the definition, we obtain

$$
\begin{aligned}
\left(u_{1}\left(\varphi_{1}-x\right)_{+}\right)_{t}-\left(u_{2}\left(\varphi_{2}-x\right)_{+}\right)_{t}+\left(u_{1}^{2}\left(\varphi_{1}-x\right)_{+}^{2}\right)_{x}+\left(u_{2}^{2}\left(\varphi_{2}-x\right)_{+}^{2}\right)_{x} \\
\quad+2\left[u_{0} u_{1}\left(\varphi_{1}-x\right)_{+}\right]_{x}-2\left[u_{0} u_{2}\left(\varphi_{2}-x\right)_{+}\right]_{x} \\
\quad-2\left[u_{1} u_{2}\left(\varphi_{1}-x\right)\left(\varphi_{2}-x\right) \theta\left(\varphi_{1}-x\right)\right]_{x} B_{1}\left(\frac{\Delta \varphi}{\varepsilon}\right) \\
\quad-2\left[u_{1} u_{2}\left(\varphi_{1}-x\right)\left(\varphi_{2}-x\right) \theta\left(\varphi_{2}-x\right)\right]_{x} B_{2}\left(\frac{\Delta \varphi}{\varepsilon}\right)=0, \quad \Delta \varphi=\varphi_{2}-\varphi_{1} .
\end{aligned}
$$




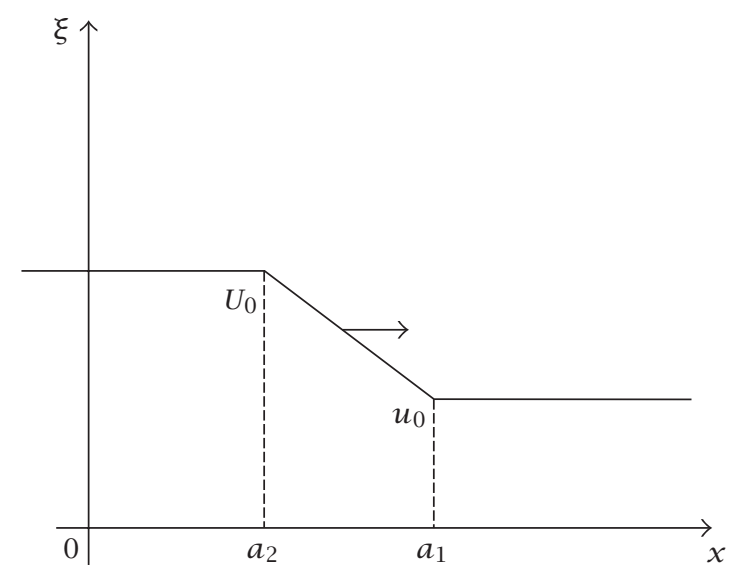

FIGURE 3.1

Consider the domain $\varphi_{2}<x \leq \varphi_{1}$. We obtain

$$
\begin{aligned}
& u_{1 t}\left(\varphi_{1}-x\right)+u_{1} \varphi_{1 t}+2\left[u_{0} u_{1}\left(\varphi_{1}-x\right)\right]_{x} \\
& \quad-\left[u_{1}^{2}\left(\varphi_{1}-x\right)^{2}\right]_{x}+2 u_{1} u_{2}\left(\varphi_{1}-x\right) B_{1}+2 u_{1} u_{2}\left(\varphi_{2}-x\right) B_{2}=0 .
\end{aligned}
$$

We set $x=\varphi_{1}$. Since we have $\partial u_{i} / \partial x \equiv 0$ in our example, we obtain

$$
u_{1} \varphi_{1 t}-2 u_{0} u_{1}+2 u_{1} u_{2} \Delta \varphi B_{1}=0 .
$$

Substituting (3.18) into (3.17), we arrive at the following equation for the function $u_{1}$ :

$$
u_{1 t}-2 u_{1}^{2}+4 u_{1} u_{2} B_{1}=0 .
$$

In a similar way, considering the domain $-\infty<x \leq \varphi_{2}$, we obtain the other two equations

$$
\begin{aligned}
\varphi_{2 t}-2 u_{0}+2 u_{1} \Delta \varphi B_{2}\left(\frac{\Delta \varphi}{\varepsilon}\right) & =0, \\
u_{2 t}+2 u_{2}^{2}-4 u_{1} u_{2} B_{2}\left(\frac{\Delta \varphi}{\varepsilon}\right) & =0, \quad \Delta \varphi=\varphi_{2}-\varphi_{1} .
\end{aligned}
$$

Let $\Delta \varphi<0$, then, up to $O\left(\varepsilon^{N}\right)$, we have $B_{1}(\Delta \varphi / \varepsilon)=0, B_{2}(\Delta \varphi / \varepsilon)=1$ and obtain the following system of equations describing the evolution of the broken line until it turns over:

$$
\begin{aligned}
\left(\varphi_{10}\right)_{t}^{\prime}-2 u_{0} & =0, & \left(\varphi_{20}\right)_{t}^{\prime}-2 u_{0}+2 u_{10}\left(\varphi_{20}-\varphi_{10}\right)=0, \\
\left(\varphi_{10}\right)_{t}^{\prime}-2\left(u_{10}\right)^{2} & =0, & \left(u_{20}\right)_{t}^{\prime}+2 u_{20}^{2}-4 u_{10} u_{20}=0 .
\end{aligned}
$$

Solutions of this system have the form

$$
\begin{gathered}
u_{10}(t)=u_{20}(t)=\frac{u_{1}^{0}}{1-2 t u_{1}^{0}}, \\
\varphi_{10}=a_{1}+2 u_{0} t, \quad \varphi_{20}=a_{2}+2\left[u_{1}^{0}\left(a_{1}-a_{2}\right)+u_{0}\right] t .
\end{gathered}
$$

We write $\psi_{0}=\varphi_{20}(t)-\varphi_{10}(t)$. At time $t=t^{*}$ such that $\psi_{0}\left(t^{*}\right)=0$ the weak discontinuities merge and a shock wave is generated. 
To construct formulas that are uniform in $t$ and describe the confluence of weak discontinuities and the generation of a shock wave, we seek the solution of (3.21) in the form

$$
\varphi_{k}(t, \varepsilon)=\varphi_{k 0}(t)+\psi_{0} \phi_{k 1}(\tau), \quad \tau=\frac{\psi_{0}}{\varepsilon},
$$

where the functions $\phi_{k 1}(\tau)$ satisfy the same conditions as in Section 3.1.

We seek the functions $u_{\varepsilon}(t, \varepsilon)$ in the form

$$
u_{k}(t, \varepsilon)=\frac{\psi_{0}(0) u_{1}^{0}}{\psi_{0}+\varepsilon g_{k}(\tau)} .
$$

Here we assume that the functions $g_{k}(\tau)$ behave in the same way as the functions $\phi_{k 1}(\tau)$ and take into account the relation

$$
\frac{u_{10}}{u_{1}^{0}}=\frac{1}{1-2 t u_{1}^{0}}=\frac{\psi_{0}(0)}{\psi_{0}(t)}
$$

follows from the equation $\psi_{0 t}+2 u_{10} \psi_{0}=0$. After simple calculations we see that the function $g=g_{1}=g_{2}$ satisfies the equation $\dot{\mathfrak{g}}+2\left(1-B_{2}(\rho)\right)=0$ and the function $\rho=\rho(\tau)=\Delta \varphi / \varepsilon$ is a solution of the boundary problem

$$
\dot{\rho}=1-2 B_{1}(\rho),\left.\quad \frac{\rho}{\tau}\right|_{\tau \rightarrow-\infty} \longrightarrow 1 .
$$

As before, the equation $\dot{\rho}=1-2 B_{1}(\rho)$ has a single root $\rho=\rho_{0}$ such that $B_{1}\left(\rho_{0}\right)=$ $B_{2}\left(\rho_{0}\right)=1 / 2$ and $\rho \rightarrow \rho_{0}$ as $\tau \rightarrow \infty$. This allows us to calculate the solution for $\Delta \psi_{0}>0$ (i.e., after the interaction) or as $\tau \rightarrow \infty$.

We introduce the function $G(\tau)=\tau+g(\tau)$. Obviously, $\dot{G}=\dot{\rho}, G /\left.\tau\right|_{\tau \rightarrow-\infty} \rightarrow+1$, and we choose

$$
G=-\int_{-\infty}^{\infty}\left(1-2 B_{1}(\rho)\right) d \tau^{\prime}+\rho_{0} .
$$

On the other hand, we can express the functions $u_{i}$ via the function $G$

$$
u_{i}=\frac{\psi_{0}(0) u_{1}^{0}}{\varepsilon G} \stackrel{\tau \rightarrow \infty}{\longrightarrow} \frac{\psi_{0}(0) u_{1}^{0}}{\varepsilon \rho_{0}} .
$$

We calculate the limit $\left(\varphi_{k}\right)_{t}^{+}$as $\tau \rightarrow \infty$ of the velocities of the weak discontinuities

$$
\begin{aligned}
& \left(\varphi_{2}\right)_{t}^{+}=2 u_{0}-\frac{2 \psi_{0}(0) u_{1}^{0}}{\varepsilon \rho_{0}} \frac{1}{2} \varepsilon \rho_{0}=2 u_{0}+\left(a_{1}-a_{2}\right) u_{1}^{0}, \\
& \left(\varphi_{1}\right)_{t}^{+}=2 u_{0}-\frac{2 \psi_{0}(0) u_{1}^{0}}{\varepsilon \rho_{0}} \frac{1}{2} \varepsilon \rho_{0}=2 u_{0}+\left(a_{1}-a_{2}\right) u_{1}^{0},
\end{aligned}
$$

which coincides with the velocity of the shock wave

$$
U(x, t)=u_{0}+\left(a_{1}-a_{2}\right) u_{1}^{0} \theta\left(-x+\varphi^{+}(t)\right),
$$

where $\varphi^{+}=\left(\varphi_{2}^{+}\right)_{t}=\left(\varphi_{1}^{-}\right)_{t}$. By using the explicit formula for the solution $u_{\varepsilon}(x, t)$, we can easily show that

$$
w-\lim _{\varepsilon \rightarrow 0} u_{\varepsilon}(x, t)=U(x, t), \quad t>t^{*} .
$$


To this end, we rewrite the above-constructed solution $u_{\varepsilon}(x, t)$ in the form

$$
\begin{aligned}
u_{\varepsilon}(x, t)= & u_{0}+u_{1}\left(\varphi_{1}-\varphi_{2}\right) \theta_{\varepsilon, 1}\left(\varphi_{1}-x\right) \\
& +u_{1}\left(x-\varphi_{2}\right)\left[\theta_{\varepsilon, 2}\left(\varphi_{2}-x\right)-\theta_{\varepsilon, 1}\left(\varphi_{1}-x\right)\right] .
\end{aligned}
$$

Consider the second term. We have

$$
u_{1}\left(\varphi_{1}-\varphi_{2}\right)=\frac{\psi_{0}(0) u_{1}^{0} \rho}{G}=\psi_{0}(0) u_{1}^{0}=\left(a_{1}-a_{2}\right) u_{1}^{0} \stackrel{\text { def }}{=} U_{0} .
$$

Since $\left.\varphi_{1}\right|_{t>t^{*}} \simeq \varphi^{+}$, the first two terms pass into the shock wave $U(x, t)$ for $t>t^{*}$. Consider the last term

$$
\begin{aligned}
u_{1}\left(x-\varphi_{2}\right) & {\left[\theta_{\varepsilon, 2}\left(\varphi_{2}-x\right)-\theta_{\varepsilon, 1}\left(\varphi_{1}-x\right)\right] } \\
= & u_{1}\left(\varphi_{1}-\varphi_{2}\right)\left[\frac{\theta_{\varepsilon, 2}\left(\varphi_{2}-x\right)-\theta_{\varepsilon, 1}\left(\varphi_{1}-x\right)}{\varphi_{1}-\varphi_{2}}\right]\left(x-\varphi_{2}\right) .
\end{aligned}
$$

As was already shown, the coefficient of the expression in front of braces is a constant. The expression in square brackets is an approximation of the $\delta$-function at the point $\varphi_{2}$. Hence the entire expression in braces is small (in a uniform metric) as $\varepsilon \rightarrow 0$.

We study the problem in which a shock wave is generated by a special (piecewise linear) initial condition. The case of a general smooth initial functions can be treated similarly. Here we need to consider a family of linear interpolations of this initial condition and to use the above technique on segments of the broken line.

To study this problem in more detail, we note that we have considered only one possibility of evolution of the broken line, namely, formation of a step. Another mechanism of evolution is as follows: segments of the broken line are added to the step that has already been formed. This is the confluence of a weak discontinuity and $a$ shock wave.

Now we again consider the Hopf equation in order to study this mechanism. The initial condition corresponding to this type of interaction has the form

$$
\left.u\right|_{t=0}=u_{0}^{0} \theta\left(a_{1}^{0}-x\right)+u_{1}^{0}\left(a_{1}-x\right) \theta\left(a_{1}-x\right)-u_{1}^{0}\left(a_{2}-x\right) \theta\left(a_{2}-x\right),
$$

where $u_{0}^{0}, u_{1}^{0}$ are positive constants and $a_{1}>a_{2}$ (see Figure 3.2).

Just as before, we construct the approximation of the solution in the form

$$
u_{\varepsilon}(x, t)=u_{0} \theta_{\varepsilon, 1}\left(\varphi_{1}-x\right)+u_{1}\left(\varphi_{1}-x\right) \theta_{\varepsilon, 1}\left(\varphi_{1}-x\right)-u_{1}\left(\varphi_{2}-x\right) \theta_{\varepsilon, 2}\left(\varphi_{2}-x\right),
$$

where $u_{i}=u_{i}(t, \varepsilon), \varphi_{i}=\varphi_{i}(t, \varepsilon)$. Substituting this expression into the Hopf equation, we obtain the system of equations (cf. (3.18), (3.19), and (3.20))

$$
\begin{aligned}
\varphi_{1 t}-u_{0}+2 u_{1} \psi B_{1} & =0, & & u_{1 t}-2 u_{1}^{2}+4 u_{1}^{2} B_{1}=0, \\
\varphi_{2 t}-2 u_{0} B_{2}+2 u_{1} \psi B_{2} & =0, & & u_{0 t}-u_{0} u_{1}\left(1-2 B_{1}\right)=0,
\end{aligned}
$$

where $B_{i}=B_{i}(\Delta \varphi / \varepsilon)$ are the functions derived above, $\Delta \varphi=\varphi_{2}-\varphi_{1}$. Before the interaction, we have $\varphi_{2}<\varphi_{1}, \Delta \varphi / \varepsilon \sim-\infty$, and $B_{1}=0, B_{2}=1$ with arbitrary accuracy in $\varepsilon$. Denoting by $\varphi_{10}, u_{10}, \varphi_{20}, u_{00}$ the solution of system (3.37) with $B_{1}=0$, $B_{2}=1$, we obtain the following system of equations for these functions:

$$
\begin{aligned}
& \varphi_{10 t}=u_{00}, \quad u_{10 t}=2 u_{10}^{2}, \quad u_{00 t}=u_{00} u_{10}, \\
& \varphi_{20 t}=2\left(u_{00}-u_{10} \psi_{0}\right), \quad \psi_{0}=\varphi_{20}-\varphi_{10} .
\end{aligned}
$$




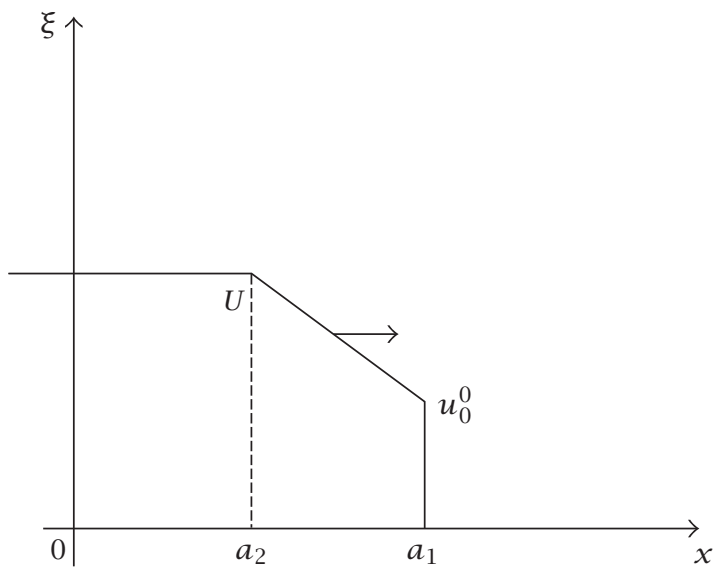

FIGURE 3.2

It is easy to find the solution of this system

$$
\begin{aligned}
u_{10} & =\frac{u_{1}^{0}}{1-2 u_{1}^{0} t}, \quad u_{00}=\frac{u_{0}^{0}}{\left(1-2 u_{1}^{0} t\right)^{1 / 2}}, \\
\varphi_{20} & =a_{2}+2 U t, \quad \varphi_{10}=a_{1}+\int_{0}^{t} u_{00} d t, \\
\psi_{0} & =\frac{1}{u_{1}^{0}}\left[\left(\psi_{0}^{0} u_{1}^{0}-u_{0}^{0}\right)\left(1-2 u_{0}^{1} t\right)-u_{0}^{0} \sqrt{1-2 u_{0}^{1} t}\right], \\
U & =u_{0}^{0}+u_{1}^{0}\left(a_{1}-a_{2}\right) .
\end{aligned}
$$

One can easily see that the function $\psi_{0}(t)$ vanishes at the two points $t_{1}=1 / 2 u_{0}^{1}$ and $t^{*}$ such that

$$
\sqrt{1-2 u_{0}^{1} t^{*}}=\frac{u_{0}^{0}}{U}
$$

Obviously, $t^{*}<t_{1}$ and the free singularities supports $x=\varphi_{10}$ and $x=\varphi_{20}$ merge at $t=t^{*}$. In this case we have

$$
u_{00}\left(t^{*}\right)=u_{00}^{*} \equiv U, \quad u_{10}\left(t^{*}\right)=\frac{U^{2}}{\left(u_{0}^{0}\right)^{2}} u_{1}^{0}<\infty .
$$

Thus in this example the mechanism of formation of a new shock wave consists not in turning over the inclined segment of the broken line, as in the preceding example, but in the disappearance of this inclined segment due to increasing vertical segment.

Subtracting the first equation from the third equation in (3.37), we obtain the following equation for the function $\psi$ :

$$
\psi_{t}=\left(u_{0}-2 \psi u_{1}\right)\left(1-2 B_{1}\left(\frac{\Delta \varphi}{\varepsilon}\right)\right)
$$

or, denoting $\rho=\Delta \varphi / \varepsilon=\left(\psi_{0}+\psi_{0} \psi_{1}(\tau)\right) / \varepsilon, \tau=\psi_{0} / \varepsilon$,

$$
\psi_{0}^{\prime} \dot{\rho}=\left[u_{0}-2 \psi u_{1}\right]\left(1-2 B_{1}(\rho)\right),\left.\quad \frac{\rho}{\tau}\right|_{\tau \rightarrow-\infty} \longrightarrow 1 \text {. }
$$


Note that we can use the formula for $\psi_{0}$ (and for the functions $u_{00}$ and $u_{10}$ ) only for $t \in\left[0, t^{*}+\delta\right]$, where $\delta>0$ is any number such that $\delta<t_{1}-t^{*}$.

To obtain formulas that are global in $t$, we need to choose a number $\delta$ and continue the functions $u_{00}, u_{10}$, and $\psi_{0}$ smoothly to the time $t \geq t^{*}+\delta$ so that the sign is preserved. Calculating the coefficient of $u_{0}-2 \psi u_{1}$, one can easily see that $\rho>0$ for $t<t^{*}$. Hence there exists a solution $\rho \rightarrow \rho_{0}$, where $\rho_{0}$ is a root of the equation

$$
B_{1}(\rho)=\frac{1}{2} .
$$

Consider the system of equations for the functions $u_{0}$ and $u_{1}$. By the change $u_{0}=$ $u_{0}^{0} \sqrt{u_{1} / u_{1}^{0}}$, this system can be reduced to the single equation for $u_{1}$ :

$$
u_{1 t}-2 u_{1}^{2}\left(1-2 B_{1}\right)=0 .
$$

Its solution has the form

$$
u_{1}(t, \varepsilon)=\frac{u_{1}^{0}}{1-2 u_{1}^{0} \int_{0}^{t}\left[1-2 B_{1}(\rho(\tau))\right] d t^{\prime}} .
$$

Clearly, we have $u_{1}\left(t^{*}, \varepsilon\right) \leq u_{10}\left(t^{*}\right)$ (since $\left.\int_{0}^{t^{*}}\left(1-2 B_{1}\right) d t^{\prime} \leq t^{*}\right)$. On the other hand, we have $t>t^{*}$ for $\rho \rightarrow \rho_{0}$. Therefore, $\psi_{1}(\tau) \rightarrow-1$ as $\tau \rightarrow \infty$ and hence $(\Delta \varphi) \rightarrow 0$ as $\tau \rightarrow \infty$ (i.e., for $t>t^{*}, \varepsilon \rightarrow 0$ ). This implies that for $t>t^{*}$ we have

$$
u_{1}(t, \varepsilon)=u_{10}\left(t^{*}\right)+o(1), \quad \varepsilon \longrightarrow 0 .
$$

We represent the above-constructed solution in the form

$$
\begin{aligned}
u_{\varepsilon}(x, t)= & U \theta_{\varepsilon, 1}\left(\varphi_{1}-x\right)+\left(u_{0}-U\right)\left[\theta_{\varepsilon, 1}\left(\varphi_{1}-x\right)-\theta_{\varepsilon, 2}\left(\varphi_{2}-x\right)\right]\left(\varphi_{2}-x\right) \\
& +\left[\theta_{\varepsilon, 1}\left(\varphi_{1}-x\right)-\theta_{\varepsilon, 2}\left(\varphi_{2}-x\right)\right]\left(\varphi_{2}-x\right) u_{1} .
\end{aligned}
$$

Obviously, for $t>t^{*}, \varepsilon \rightarrow 0$, the first term approximates the shock wave

$$
u=U \theta\left(a_{1}+U t-x\right)
$$

and in this case the second term vanishes since $u_{0}-U \rightarrow 0$ and the third term vanishes since $\varphi_{1}-\varphi_{2}=\Delta \varphi \rightarrow 0$. Recall that $\psi_{0 t}=u_{00}-2 \psi_{0} u_{10}$ for $t<t^{*}$. In view of (3.42), we can continue the function $\psi_{0 t}$ for $t>t^{*}$ in the form $\psi_{0 t}=U$. In this case the function $u_{0}-2 \psi u_{1}$ is continuous uniformly in $\varepsilon$ for $t=t^{*}$ and we can show that the function $\rho$ is a solution of the boundary value problem

$$
\dot{\rho}=\left(1-2 B_{1}(\rho)\right),\left.\quad \frac{\rho}{\tau}\right|_{\tau \rightarrow-\infty} \longrightarrow 1 .
$$

The system of equations determining the weak asymptotic solution in this case also splits into separate equations.

Now we briefly consider the problem of decay of nonstable shock waves.

One can easily see that by setting $v_{T, \varepsilon}(x, t)=u_{\varepsilon}(x, T-t), T>t^{*}$, we obtain a $T$-dependent family of weak asymptotic solutions of the equation $v_{t}-\left(v^{2}\right)_{x}=0$. 
For $t=0$ the solutions of this family are shock waves (unstable for this new equation). The weak limit of these solutions for $0 \leq t<T-t^{*}$ is a shock wave, and for $t>T-t^{*}$ is a broken line consisting of two moving weak discontinuities into which the unstable shock wave splits at time $t_{*}(T)=T-t^{*}$ (which is not unique).

3.3. Interaction of shock waves in the multidimensional case. Consider the twodimensional nonlinear equation arising in the reservoir problem

$$
L[u]=\frac{\partial u}{\partial t}+A_{1} \frac{\partial u^{2}}{\partial x_{1}}+A_{2} \frac{\partial u^{2}}{\partial x_{2}}=0 .
$$

The above approach can be easily generalized to the case of an arbitrary dimension if the codimension of the front of the nonlinear wave is 1 . We assume that $A_{1} \neq A_{2}$ are positive constants.

We choose the initial conditions as

$$
\left.u\right|_{t=0}=u_{0}+u_{1} \theta\left(t+\psi_{1}(x)\right)+u_{2} \theta\left(t+\psi_{1}(x)\right)
$$

where $x=\left(x_{1}, x_{2}\right), u_{i}$ are positive constants, and $\psi_{i}(x)$ are the desired functions. We write $\Gamma_{i}^{0}=\left\{x, \psi_{i}(x)=0\right\}$.

Clearly, the curves $\Gamma_{i}^{0}$ are given initial positions of the fronts of two shock waves whose sum is just the initial condition. In addition, we assume that the curves $\Gamma_{i}^{0}$ are transversal to the vector field $\langle\vec{A}, \nabla\rangle, \vec{A}=\left(A_{1}, A_{2}\right)$ and $\Gamma_{2}^{0}$ is the cross-section of the (trivial) fibration over $\Gamma_{1}^{0}$ whose fibers are straight lines parallel to the vector $\vec{A}$. In addition, we assume that the motion from the points of $\Gamma_{2}^{0}$ to the points of $\Gamma_{1}^{0}$ is in the direction of the vector $\vec{A}$. In this case the fact that $u_{1}, u_{2}$ are positive constants is a sufficient condition of stability.

If the functions $\psi_{i}(x)$ are known, then the curves (level surfaces) $\Gamma_{i}^{t}\left\{x, t+\psi_{i}(x)=0\right\}$ determine the fronts of shock waves at time $t$.

Acting as before (see Section 3.1), we substitute the approximation

$$
u_{\varepsilon}(x, t)=u_{0}+u_{1} \theta_{\varepsilon, 1}\left(t+\psi_{1}(x, \varepsilon)\right)+u_{2} \theta_{\varepsilon, 2}\left(t+\psi_{2}(x, \varepsilon)\right)
$$

into (3.51) and calculate the weak asymptotics of $L\left[u_{\varepsilon}\right]$. We obtain

$$
\begin{gathered}
L\left[u_{\varepsilon}\right]=\delta_{\Gamma_{1}^{t}}\left[1+\left\langle\vec{A}, \nabla \psi_{1}\right\rangle\left(u_{1}+2 u_{0}+2 u_{2} B_{1}\left(\frac{\Delta \psi}{\varepsilon}\right)\right)\right] \\
+\delta_{\Gamma_{2}^{t}}\left[1+\left\langle\vec{A}, \nabla \psi_{2}\right\rangle\left(2 u_{0}+u_{2}+2 u_{1} B_{2}\left(\frac{\Delta \psi}{\varepsilon}\right)\right)\right]+O_{\mathscr{P}^{\prime}}(\varepsilon), \\
\Delta \psi=\psi_{2}-\psi_{1} .
\end{gathered}
$$

Here the functions $B_{1}$ and $B_{2}$ are the same as in Section 3.1. Formulas for the weak asymptotics in the multidimensional case are carried out in the same way as in the one-dimensional case.

Roughly speaking, the (two-dimensional) integral becomes an iterated integral over the surface $\Gamma_{i}^{t}$ and over the normal to this surface. The asymptotics of the integral along the normal is calculated in the same way as in the one-dimensional case, see [2]. 
It follows from our assumptions that the inequality $\Delta \psi<0$ holds for sufficiently small positive $t$. Hence, we have $\Delta \psi / \varepsilon \sim-\infty$ and for small $t$ we obtain the following equations describing the system of noninteracting fronts:

$$
1+\left\langle\vec{A}, \nabla \psi_{1}\right\rangle\left(u_{1}+2 u_{0}\right)=0, \quad 1+\left\langle\vec{A}, \nabla \psi_{2}\right\rangle\left(u_{2}+2 u_{1}+2 u_{0}\right)=0 .
$$

Clearly, these equations determine the (limit) functions $\psi_{k 0}$ if the curves $\Gamma_{i}^{0}$ on which they vanish are given.

Dividing these equations by $\left|\nabla \psi_{i}\right|$ and taking into account the fact that, in view of our formulas, the waves travel in the direction of decreasing functions $\psi_{k 0}(x)$, we can rewrite the last system as

$$
V_{n_{1}}^{(1)}=\left\langle\vec{A}, \vec{n}_{1}\right\rangle\left(u_{1}+2 u_{0}\right), \quad V_{n_{2}}^{(2)}=\left\langle\vec{A}, \vec{n}_{2}\right\rangle\left(u_{2}+2 u_{1}+2 u_{0}\right),
$$

where $n_{i}$ is the normal (at a point) to $\Gamma_{i}^{t}, V_{n_{i}}^{(i)}$ is the normal velocity of this point. Clearly, the velocities of the points of the curve $\Gamma_{2}^{t}$ are larger than the velocities of the points of the curve $\Gamma_{2}^{t}$ along the trajectories of the field $\langle\vec{A}, \nabla\rangle$, but the distance between the curves $\Gamma_{i}^{t}$ along the trajectory depends, in general, on the point.

Therefore, since the shape of these curves is rather arbitrary, there may be no complete confluence of these curves at their contact. A new shock wave with summary amplitude $u_{1}+u_{2}$ is generated at the points of contact. This shock wave travels with its new velocity, and the solution may be of a rather complicated structure. To describe this wave uniformly in time, we seek the solution of the system

$$
\begin{aligned}
& 1+\left\langle\vec{A}, \nabla \psi_{1}\right\rangle\left(u_{1}+2 u_{0}+2 u_{2} B_{1}\left(\frac{\Delta \psi}{\varepsilon}\right)\right)=0, \\
& 1+\left\langle\vec{A}, \nabla \psi_{2}\right\rangle\left(u_{2}+2 u_{0}+2 u_{1} B_{2}\left(\frac{\Delta \psi}{\varepsilon}\right)\right)=0
\end{aligned}
$$

in the form

$$
\psi_{k}(x, \varepsilon)=\psi_{k 0}(x)+\phi_{0}(x) \psi_{k 1}\left(\frac{\phi_{0}}{\varepsilon}\right),
$$

where $\phi_{0}=\psi_{20}(x)-\psi_{10}(x)$. Note that, in view of our assumptions on the geometry, instead of the coordinates $\left(x_{1}, x_{2}\right)$, we can introduce the coordinates $(s, \xi)$, where $s$ are the coordinates on $\Gamma_{2}^{0}$ and $\xi$ is a parameter on the trajectories of the vector field $\langle\vec{A}, \nabla\rangle$.

Hence we, in fact, "calculate the distance" between the curves $\Gamma_{i}^{+}$(i.e., the differences $\left.\Delta \psi, \phi_{0}\right)$ along the trajectories of the field $\langle\vec{A}, \nabla\rangle$.

Preserving, instead of $d / d \xi$, the notation $\langle\vec{A}, \nabla\rangle$, substituting (3.57) into (3.55), and taking into account (3.58), we obtain

$$
\begin{gathered}
2\left\langle\vec{A}, \nabla \psi_{10}\right\rangle u_{2} B_{1}+\left\langle\vec{A}, \nabla \phi_{0}\right\rangle \frac{d}{d \tau}\left(\tau \psi_{11}\right)\left[U_{0}+2 u_{2}\left(B_{1}-\frac{1}{2}\right)\right]=0, \\
-2 u_{1}\left\langle\vec{A}, \nabla \psi_{20}\right\rangle+2\left\langle\vec{A}, \nabla \psi_{20}\right\rangle u_{1} B_{2}+\left\langle\vec{A}, \nabla \phi_{0}\right\rangle \frac{d}{d \tau}\left(\tau \psi_{21}\right)\left[U_{0}+2 u_{1}\left(B_{2}-\frac{1}{2}\right)\right]=0 .
\end{gathered}
$$

Here $U_{0}=u_{1}+u_{2}+2 u_{0}, \tau=\phi_{0} / \varepsilon$. 
The further is similar to that in the one-dimensional case. Its first stage is to obtain an equation for the function $\rho=\Delta \psi / \varepsilon=\left(\phi_{0} / \varepsilon\right)\left(1+\psi_{21}(\tau)-\psi_{11}(\tau)\right) \stackrel{\text { def }}{=} \tau\left(1+\phi_{1}(\tau)\right)$. Next, we calculate the limits of the functions $B_{k}(\rho)$ as $\tau \rightarrow \infty$ (after the interaction) and find equations for the limit functions $\psi_{k}^{+}$as well as equations for $\psi_{k 1}(\tau)$.

Subtracting the first equation in (3.59) from the second one and carrying out several calculations, we obtain the desired equation for $\rho$

$$
\dot{\rho}=1-\frac{1-B_{2}(\rho)}{u_{1}+u_{2}}\left[\frac{2 u_{2} U_{2}}{U_{0}-2 u_{2}\left(B_{2}-1 / 2\right)}+\frac{2 u_{1} U_{1}}{U_{1}+2 u_{1}\left(B_{2}-1 / 2\right)}\right],\left.\quad \frac{\rho}{\tau}\right|_{\tau \rightarrow-\infty} \longrightarrow 1 .
$$

One can show that the right-hand side of this equation (that differs, as one can see, from that in the similar equation in the one-dimensional case) also has a single root $\rho_{0}$ and $B_{2}\left(\rho_{0}\right)=1 / 2$ (and hence $\left.B_{1}\left(\rho_{0}\right)=1 / 2\right)$.

Hence it follows from (3.55) that, for the same values of $s$ for which a point of the curve $\Gamma_{1}^{+}$"outruns" the curve $\Gamma_{1}^{+}$, we have

$$
1+\left\langle\vec{A}, \nabla \psi_{k}^{+}\right\rangle\left(u_{1}+u_{2}+2 u_{0}\right)=0, \quad k=1,2 .
$$

This implies that $\psi_{1}^{+}=\psi_{2}^{+}$and for a given $s$, after the confluence of the curves, a wave with summary amplitude $u_{1}+u_{2}$ travels in the direction of $\vec{A}$. Thus, for a fixed $s$, the dynamics of interaction in the direction of $\vec{A}$ is similar to that in the onedimensional case.

Here we do not write out the equations for $\psi_{k 1}$. They can be obtained in the same way as the similar equations in the one-dimensional case.

4. Conclusion. The problem of shock wave interaction in the one-dimensional case is presented in $[3,4,5]$ not only for the Hopf equations but also for equations with sufficiently general nonlinearity. The formulas from Section 2 are derived there in more detail.

Similarly, in the multidimensional case one can easily generalize our construction to the case of more general nonlinearities, variable coefficients and amplitudes.

For reasons of space, here we do not consider the problem of constructing definitions of weak solutions. This problem is discussed in [1, 2, 4]. In particular, in [4] a definition of a weak solution is constructed for KdV type equations admitting the zero dispersion limit for soliton type solutions.

ACKNOWLEDGMENT. This work was supported by the Russian Foundation for Basic Research under grant No. 99-01-01074.

\section{REFERENCES}

[1] V. G. Danilov, A new definition of weak solutions of semilinear equations with a small parameter, Uspekhi Mat. Nauk 51 (1997), no. 5, 184 (Russian), translated in Russian Math. Surveys.

[2] V. G. Danilov, G. A. Omel'yanov, and E. V. Radkevich, Hugoniot-type conditions and weak solutions to the phase-field system, European J. Appl. Math. 10 (1999), no. 1, 55-77.

[3] V. G. Danilov and V. M. Shelkovich, Propagation and interaction of nonlinear waves, Proceedings of 8th International Conference on Hyperbolic Problems Theory, Numerics, Applications, University of Magdeburg, Magdeburg, 2000, pp. 326-328. 
[4] _ Propagation of infinitely narrow $\delta$-solitons, http://arxiv.org/abs/math-ph/0012 002, 2000.

[5] _ Propagation and interaction of shock waves of quasilinear equation, Nonlinear Stud. 8 (2001), no. 1, 135-169.

V. G. Danilov: Moscow State Institute of Electronics and Mathematics, Technical UNIVERSITY, 109028, MOSCOW, RUSSIA

E-mail address: pm@mi em. edu. ru 


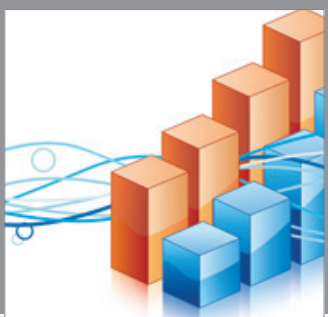

Advances in

Operations Research

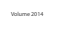

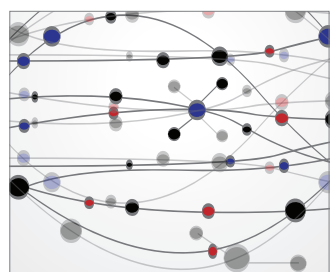

\section{The Scientific} World Journal
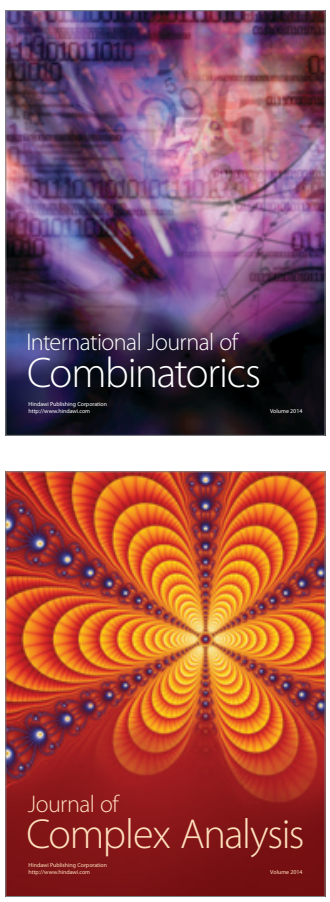

International Journal of

Mathematics and

Mathematical

Sciences
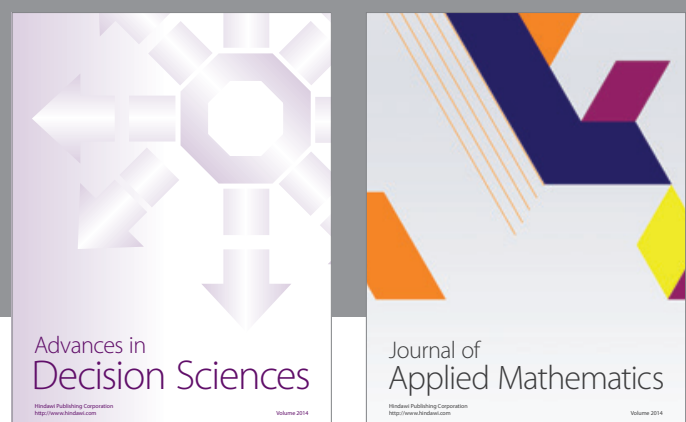

Journal of

Applied Mathematics
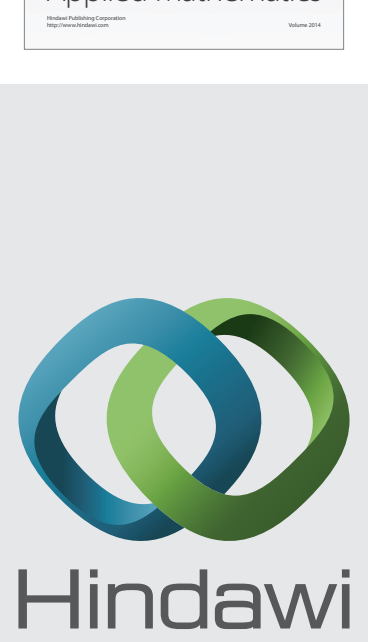

Submit your manuscripts at http://www.hindawi.com
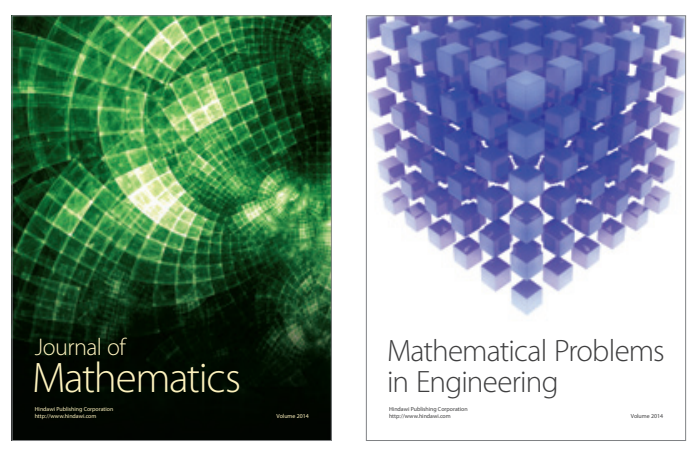

Mathematical Problems in Engineering
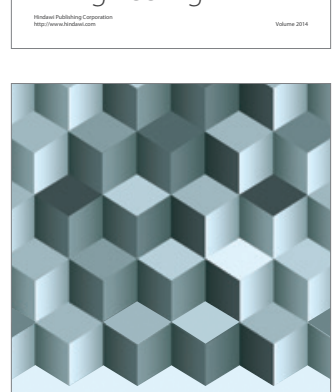

Journal of

Function Spaces
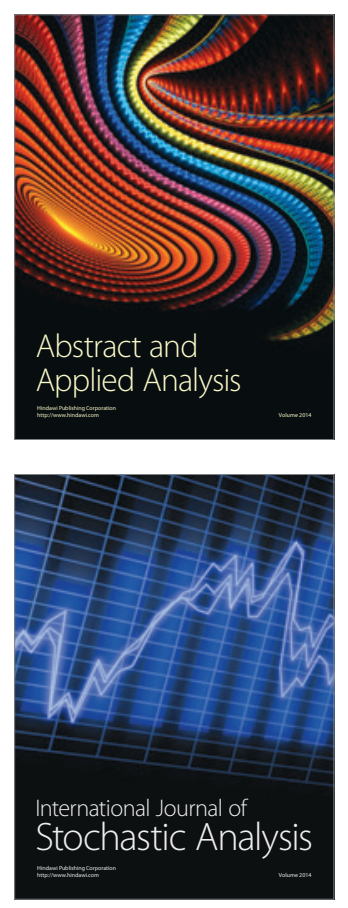

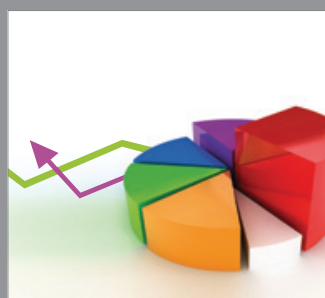

ournal of

Probability and Statistics

Promensencen
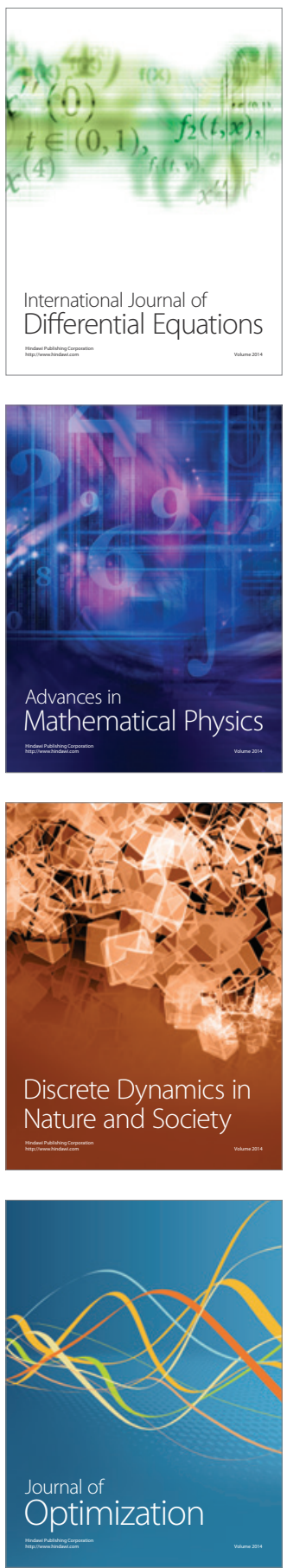